\title{
Some bounds on local connective Chromatic number
}

\author{
Canan Ciftci and Pinar Dundar \\ Department of Mathematics, Faculty of Science, Ege University,35100, Izmir/Turkey
}

Received: 6 February 2017, Accepted: 13 May 2017

Published online: 14 August 2017.

\begin{abstract}
Graph coloring is one of the most important concept in graph theory. Many practical problems can be formulated as graph coloring problems. In this paper, we define a new coloring concept called local connective coloring. A local connective $k$-coloring of a graph $G$ is a proper vertex coloring, which assigns colors from $\{1,2, \ldots, k\}$ to the vertices $V(G)$ in a such way that any two nonadjacent vertices $u$ and $v$ of a color $i$ satisfies $\kappa(u, v) \geqslant i$, where $\kappa(u, v)$ is the maximum number of internally disjoint paths between $u$ and $v$. Adjacent vertices are colored with different colors as in the proper coloring. The smallest integer $k$ for which there exists a local connective $k$ - coloring of $G$ is called the local connective chromatic number of $G$, and it is denoted by $\chi_{l c}(G)$. We study this coloring on several classes of graphs and give some general bounds. We also compare local connective chromatic number of a graph with chromatic number and packing chromatic number of it.
\end{abstract}

Keywords: Graph coloring, packing chromatic number, internally disjoint path, local connective chromatic number.

\section{Introduction}

Graph coloring is a special case of graph labeling; it is an assignment of labels called "colors" to vertices or edges of a graph to certain constraints. Vertex coloring is a way of coloring the vertices of a graph such that no two adjacent vertices share the same color; this is called a proper coloring. The chromatic number $\chi(G)$ of $G$ is the minimum number of colors needed in a proper coloring of a graph. There are several graph coloring applications such as scheduling, timetabling, frequency allocation, wavelength routing and many more $[1,11,12,13]$.

In this paper, we introduce and study a new graph coloring concept called local connective coloring. For this coloring we are inspired by the notion of packing chromatic number. In 2008, Goddard et al.[14] establised the notion of packing chromatic number under the name broadcast chromatic number. The term packing chromatic number was introduced by Brešar et al. [4]. The concept of packing coloring comes from the area of frequency planning in wireless networks.

A packing $k$-coloring of a graph $G$ is a mapping $c: V(G) \longrightarrow\{1,2, \ldots, k\}$ such that any two vertices of color $i$ are at a distance of at least $i+1$. Thus, the vertices of $G$ are partitioned into different color classes $X_{1}, X_{2}, \ldots, X_{k}$, where every $X_{i}$ is an $i$-packing of $G$. The $i$-packing number of $G$, denoted by $\rho_{i}(G)$, is the maximum cardinality of an $i$-packing that occurs in $G$. The packing chromatic number $\chi_{p}(G)$ of $G$ is the smallest integer $k$ for which $G$ has packing $k$-coloring [3, $4,10,14]$.

A set of paths from a vertex $u$ to a vertex $v$ is said to be internally vertex-disjoint (abbreviated as internally disjoint) if no two paths share a common vertex except $u$ and $v$. Internally disjoint paths contribute to transfer information quickly and safely between any two vertices in an interconnection network and provide alternative routes in the event of vertex or edge failures. Then the more internally disjoint paths between two vertices are the better for a network $[5,6]$. Thus we use the term internally disjoint path in our coloring and color the vertices depending on the number of internally disjoint 
paths between two vertices. A graph $G$ which has a local connective $k$-coloring can be partitioned into disjoint color classes $X_{1}, X_{2}, \ldots, X_{k}$ and can be drawn as a $k$-partite graph. Thereby, the graph is partitioned into the subsets which have disjoint paths. Looking for a secure disjoint path between two vertices $u$ and $v$ in any color class $X_{i}$, we make this search with the vertices in the other color classes. This indicates that we look for disjoint paths starting from $u$ and ending to $v$ using the vertices in the other color classes. Thus, this search can be made with $V(G)-\left(\left|X_{i}\right|-2\right)$ vertices. Local connective coloring provides to facilitate the routing of non-adjacent vertices to communicate with each other.

Throughout this paper, we consider undirected, finite and simple (no loops and multiple edges) graphs. For the notations and terminology not defined here, see [7]. For a graph $G=(V(G), E(G)), V(G)$ and $E(G)$ are the sets of vertices and edges of $G$, respectively. For two vertices $u, v \in V(G), u$ and $v$ are neighbors if $u$ and $v$ are adjacent, that is, if there is an edge $e=u v$. The open neighboorhood of a vertex $v$ in $G$, denoted by $N(v)$, is the set of all vertices adjacent to $v$. The numbers $|V(G)|=n,|E(G)|=m$ and $|N(v)|=\operatorname{deg} v$ are called the order, the size of $G$ and the degree of $v$, respectively. The minimum degree $\delta(G)=\delta$ of a graph $G$ is the smallest degree of all vertices in $G$.

The connectivity $\kappa=\kappa(G)$ of a graph $G$ is the minimum number of vertices whose removal results in a disconnected or trivial graph. The local connectivity $\kappa_{G}(u, v)=\kappa(u, v)$ between two distinct vertices $u$ and $v$ of a graph $G$ is defined as the smallest number of vertices whose removal separates $u$ and $v$. By Menger's Theorem [8], $\kappa(u, v)$ equals the maximum number of internally disjoint $u-v$ paths in $G$ and $\kappa(G)=\min \{\kappa(u, v): u, v \in V(G)\}$. It is straightforward to verify that $\kappa(G) \leqslant \delta(G)$ and $\kappa(u, v) \leqslant \min \{\operatorname{deg} u, \operatorname{deg} v\}$.

For a connected graph $G$, a clique of $G$ is a complete subgraph of $G$. The clique number of a graph $G$, denoted $\omega(G)$, is the size of a largest clique. Two vertices that are not adjacent in a graph $G$ are said to be independent. The independence number $\alpha(G)$ of a graph $G$ is the maximum cardinality among the independent sets of vertices of $G$. The diameter $\operatorname{diam}(G)$ of a graph $G$ is the maximum distance between any two vertices of $G$.

\section{Some bounds on local connective Chromatic number}

Definition 1. A local connective $k$-coloring of a graph $G$ is a mapping $c: V(G) \longrightarrow\{1,2, \ldots, k\}$ such that

(i) If $u v \in E(G)$, then $c(u) \neq c(v)$, and

(ii) If $u v \notin E(G)$ and $c(u)=c(v)=i$, then $\kappa(u, v) \geqslant i$, where $\kappa(u, v)$ is the maximum number of internally disjoint paths between $u$ and $v$.

The smallest integer $k$ for which there exists a local connective $k$-coloring of $G$ is called the local connective chromatic number of $G$, and it is denoted by $\chi_{l c}(G)$.

The first condition characterizes proper coloring. Thus, every local connective coloring is a proper coloring. The vertices of $G$ are partitioned into disjoint color classes $X_{1}, X_{2}, \ldots, X_{k}$, where each color class $X_{i}$ consists of distinct vertices $u, v \in X_{i}$ such that $\kappa(u, v) \geqslant i$ and $\bigcup_{i=1}^{n} X_{i}=V(G)$. The maximum cardinality of $X_{i}$ in $G$ is denoted by $k_{i}$.

Since all vertices of complete graph $K_{n}$ are pairwise adjacent, by definition of local connective coloring we easily say $\chi_{l c}\left(K_{n}\right)=n$.

Lemma 1. If $G$ is a connected graph of order $n$ and independence number $\alpha$, then $\chi_{l c}(G) \leq 1+n-\alpha(G)$ and equality holds if $\kappa(u, v)=1$ for all $u, v \in V(G)$.

Proof. Let $S$ be a maximum independent set of $G$ and then $|S|=\alpha(G)$. Since $S$ is independent set, the vertices in $S$ can be colored with the same color (color 1). Now, consider the remaining graph $H$ with $n-\alpha(G)$ vertices. If $H$ is complete 
graph, then $\chi_{l c}(H)=n-\alpha(G)$. Otherwise $\chi_{l c}(H)<n-\alpha(G)$. Then, we have

$$
\chi_{l c}(G)=1+\chi_{l c}(H) \leq 1+n-\alpha(G) .
$$

If $\kappa(u, v)=1$ for all $u, v \in V(G)$, then no two vertices can receive the same color $i$ for $i \geqslant 2$. Furthermore, the maximum number of vertices which receives color 1 is $\alpha(G)$. Then, the proof is completed.

Lemma 2. Let $G$ be a graph of order $n$. Then $\chi_{l c}(G) \geqslant \omega(G)$.

Proof. Let $H$ be a subgraph of $G$ which has maximum clique and $|V(H)|=p \leq n$. Since all vertices of $H$ are pairwise adjacent, these vertices must be colored with $p$ different colors. Then, the graph $G$ is colored with at least $p$ colors. Since $n \geqslant p$, we obtain $\chi_{l c}(G) \geqslant \chi_{l c}(H)=\omega(G)$.

Theorem 1. Let $G$ be a connected graph with $n \geqslant 2$ vertices. Then

$$
2 \leq \chi_{l c}(G) \leq n .
$$

Proof. For any graph $G$ with $n \geqslant 2$ vertices, since $\alpha(G) \geqslant 1$ and $\omega(G) \geqslant 2$, the proof follows from Lemma 1 and Lemma 2.

Theorem 2. Let $G$ be a graph of order $n$ with $n \geqslant 2$. Then, $\chi(G) \leq \chi_{l c}(G)$.

Proof. For $n \geqslant 2$, we know that $\chi(G) \geqslant 2$ and $\chi_{l c}(G) \geqslant 2$. Let $p$ be the total number of vertices colored with color 1 and color 2. Then $(n-p)$ vertices remain uncolored. We consider the following three cases with respect to these $(n-p)$ vertices.

Case 1. Let $n-p=0$. Then, $\chi(G)=\chi_{l c}(G)=2$.

Case 2. Let $n-p=1$. Then, we color remaining one vertex with color 3. Thus, $\chi(G)=\chi_{l c}(G)=3$.

Case 3. Let $n-p \geqslant 2$ and $u, v$ be any two remaining vertices.

Case 3.1. If $u$ and $v$ are adjacent vertices, then by definition of proper coloring and local connective coloring we must color these vertices with different colors. Then, $\chi(G)=\chi_{l c}(G)$.

Case 3.2. Let $u$ and $v$ be non-adjacent vertices. For proper coloring, we color these vertices with the same color. For local connective coloring, consider the following cases.

Case 3.2.1. If $\kappa(u, v) \geqslant i$ for $i \geqslant 3$, we color these vertices with the same color $i$. Then, $\chi(G)=\chi_{l c}(G)$.

Case 3.2.2. If $\kappa(u, v)<i$ for $i \geqslant 3$, then we color these vertices with different colors. Then, this case increases local connective chromatic number. Hence, we have $\chi(G)<\chi_{l c}(G)$.

The proof is completed from these cases.

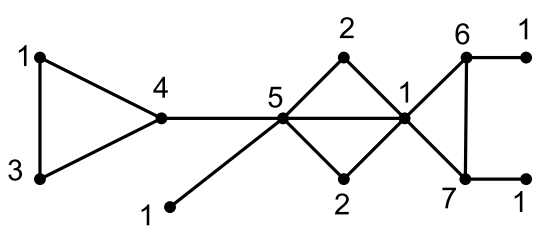

(a) $G$

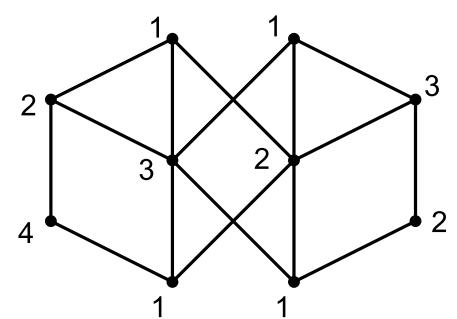

(b) $H$

Fig. 1: Local connective coloring of two graphs

Fig. 1 (a) shows the graph $G$ with $\chi_{l c}(G)>\chi_{p}(G)$ and Fig. 1 (b) shows the graph $H$ with $\chi_{l c}(H)<\chi_{p}(H)$. 
Theorem 3. Let $G$ be a graph of order $n$ with $\operatorname{diam}(G) \geqslant 2$. Then,

$$
\chi_{l c}(G) \leqslant \chi_{p}(G)+2-\operatorname{diam}(G)+\sum_{i=2}^{\operatorname{diam}(G)-1} \rho_{i}
$$

Proof. Let $G$ be a graph of order $n$, diameter $\operatorname{diam}(G) \geqslant 2$ and for $i \geqslant 1$ let $c_{i}$ be the number of vertices which is colored with packing coloring.

Since $\rho_{i}$ is the maximum number of vertices which is colored with packing coloring for $i \geqslant 1$ and $\operatorname{diam}(G) \geqslant 2$, we have $\rho_{i}(G) \leqslant 1$ for $i \geqslant \operatorname{diam}(G)$. Thereby, the graph is colored at least $\operatorname{diam}(G)-1$ colors with respect to packing coloring. For the lower bound, we give color 1 to every vertex in a maximum independent set in $G$. Then we have $c_{1}=\rho_{1}(G)=\alpha(G)$ and

$$
\begin{aligned}
\chi_{p}(G) & \geqslant \operatorname{diam}(G)-1+n-\sum_{i=1}^{\operatorname{diam}(G)-1} c_{i} \\
& =\operatorname{diam}(G)-1+n-\alpha(G)-\sum_{i=2}^{\operatorname{diam}(G)-1} c_{i}
\end{aligned}
$$

Since $c_{i} \leqslant \rho_{i}(G)$ for all $i \geqslant 1$, by inequality (1) we have

$$
\chi_{p}(G) \geqslant \operatorname{diam}(G)-1+n-\alpha(G)-\sum_{i=2}^{\operatorname{diam}(G)-1} \rho_{i} .
$$

By Lemma 1 and inequality (2), we get

$$
\chi_{l c}(G) \leqslant \chi_{p}(G)+2-\operatorname{diam}(G)+\sum_{i=2}^{\operatorname{diam}(G)-1} \rho_{i}
$$

Corollary 1. Let $G$ be a graph of order $n$ with $\operatorname{diam}(G) \geqslant 2$ and independence number $\alpha$. Then,

$$
\chi_{l c}(G) \leqslant \chi_{p}(G)+(2-\operatorname{diam}(G))(1-\alpha(G)) .
$$

Proof. Since $\rho_{i}(G) \leqslant \alpha(G)$ for $i \geqslant 2$, by Theorem 3,

$$
\begin{aligned}
\chi_{p}(G) & \geqslant \chi_{l c}(G)+\operatorname{diam}(G)-2-\sum_{i=2}^{\operatorname{diam}(G)-1} \alpha(G) \\
& =\chi_{l c}(G)+(\operatorname{diam}(G)-2)-(\operatorname{diam}(G)-2) \alpha(G) \\
& =\chi_{l c}(G)+(\operatorname{diam}(G)-2)(1-\alpha(G)) .
\end{aligned}
$$

Corollary 2. If $G$ is a graph of diameter two, then $\chi_{l c}(G) \leqslant \chi_{p}(G)$.

Proof. If we substitute $\operatorname{diam}(G)=2$ into Corollary 1, the proof is completed.

Corollary 3. If $G$ is a bipartite graph of diameter 3 , then $\chi_{l c}(G) \leqslant \chi_{p}(G)+1$.

Proof.Since $\operatorname{diam}(G)=3$, for packing coloring each color at least 3 appears at most once and since the graph is bipartite graph of diameter 3, color 2 can be used only twice [14]. Substituting $\operatorname{diam}(G)=3$ and $c_{2}=\rho_{2}(G)=2$ into the inequality of Theorem 3, we get $\chi_{l c}(G) \leqslant \chi_{p}(G)+1$. 


\section{Local connective Chromatic number of some graphs}

Theorem 4. Let $P_{n}$ be a path of order $n$ with $n \geqslant 2$. Then,

$$
\chi_{l c}\left(P_{n}\right)=1+\left\lfloor\frac{n}{2}\right\rfloor
$$

Proof. The number of internally disjoint paths between all pairs of vertices of $P_{n}$ is 1 . Then,

$$
k_{i}\left(P_{n}\right) \leq 1 \text { for } i \geqslant 2
$$

Let $X_{1}$ be a maximum independent set of $P_{n}$. Then, every vertex in $X_{1}$ has color 1 and $k_{1}\left(P_{n}\right)=\alpha\left(P_{n}\right)=\left\lceil\frac{n}{2}\right\rceil$. Since $k_{i}\left(P_{n}\right) \leq 1$ for $i \geqslant 2$, the other vertices receive different colors. Consequently, we have

$$
\chi_{l c}\left(P_{n}\right)=1+n-\left\lceil\frac{n}{2}\right\rceil=1+\left\lfloor\frac{n}{2}\right\rfloor .
$$

Theorem 5. Let $C_{n}$ be a cycle of order $n$ with $n \geqslant 3$. Then,

$$
\chi_{l c}\left(C_{n}\right)= \begin{cases}2, & \text { if } n \text { is even } \\ 3, & \text { if } n \text { is odd }\end{cases}
$$

Proof. Since cycle graphs are 2-regular graphs, $\kappa(u, v)=2$ for any two vertices $u$ and $v$ in $C_{n}$. Thus, $k_{i}\left(C_{n}\right) \leq 1$ for $i \geqslant 3$. Let $X_{1}$ and $X_{2}$ be subsets of $C_{n}$ containing the vertices labelled by color 1 and color 2 , respectively. Since $k_{i}\left(C_{n}\right) \leq 1$ for $i \geqslant 3$, the other vertices receive different colors. Therefore,

$$
k_{1}\left(C_{n}\right)=\left\lfloor\frac{n}{2}\right\rfloor, k_{2}\left(C_{n}\right)=\left\lfloor\frac{n}{2}\right\rfloor .
$$

Then, we have

$$
\begin{aligned}
\chi_{l c}\left(C_{n}\right) & =1+1+n-\left\lfloor\frac{n}{2}\right\rfloor-\left\lfloor\frac{n}{2}\right\rfloor \\
& =2+n-2\left\lfloor\frac{n}{2}\right\rfloor .
\end{aligned}
$$

The proof is completed depending on $n$ being odd and even.

Theorem 6. Let $W_{n}$ be a wheel graph of order $n$. Then,

$$
\chi_{l c}\left(W_{n}\right)= \begin{cases}3, & \text { if } n \text { is odd } \\ 4, & \text { if } n \text { is even }\end{cases}
$$

Proof. In the wheel graph $W_{n}$, the center vertex is adjacent to each vertex of the cycle $C_{n-1}$. Then this vertex must be colored with a different color than the others. Therefore, we have

$$
\chi_{l c}\left(W_{n}\right)=1+\chi_{l c}\left(C_{n-1}\right)
$$

By equality (3),

$$
\begin{aligned}
\chi_{l c}\left(W_{n}\right) & =1+2+n-1-2\left\lfloor\frac{n-1}{2}\right\rfloor \\
& =2+n-2\left\lfloor\frac{n-1}{2}\right\rfloor .
\end{aligned}
$$

The proof is completed depending on $n$ being odd and even.

Theorem 7. Let $K_{1, n}$, where $n>1$, be a star. Then, 


$$
\chi_{l c}\left(K_{1, n}\right)=2
$$

Proof. Since $\kappa(u, v) \leq \min \{\operatorname{deg} u, \operatorname{deg} v\}=1$ for any two non-adjacent vertices $u$ and $v$ of graph $K_{1, n}$, we have $k_{i}\left(K_{1, n}\right) \leq 1$ for $i \geqslant 2$. Thus, $n$ leaves of $K_{1, n}$ must be colored with color 1 and internal vertex receives a distinct color. Hence, we have $\chi_{l c}\left(K_{1, n}\right)=1+1=2$.

Theorem 8. Let $T$ be a tree of order $n$ with $n \geqslant 2$. Then

$$
\chi_{l c}(T)=1+n-\alpha(T)
$$

Proof. Let $S$ be a maximum independent set in $T$. We can give color 1 to every vertex in $S$. Thus $k_{1}(T)=\alpha(T)$. Since the number of internally disjoint paths between all non-adjacent pairs of vertices of $T$ is 1 , we have $k_{i}(T) \leq 1$ for $i \geqslant 2$. Hence, the remaining $n-\alpha(T)$ vertices receive $n-\alpha(T)$ different colors other than color 1 . Then the proof is completed.

Corollary 4. Let $T$ be a tree of order $n$ with $n \geqslant 2$. Then

$$
\chi_{l c}(T) \leq 1+\left\lfloor\frac{n}{2}\right\rfloor
$$

and equality holds if $T$ is a path.

Proof. If $T$ is a tree with $n \geqslant 2$ vertices, $\alpha(T) \geqslant\left\lceil\frac{n}{2}\right\rceil$ [2]. By Theorem 8 , we have

$$
\chi_{l c}(T)=1+n-\alpha(T) \leq 1+n-\left\lceil\frac{n}{2}\right\rceil=1+\left\lfloor\frac{n}{2}\right\rfloor .
$$

Then the proof is completed.

The complete $p$-partite graph $K_{n_{1}, n_{2}, \ldots, n_{p}}$ has a vertex set $V$ that can be partitioned into $p$ parts $V_{1}, V_{2}, \ldots, V_{p}$ so that $V_{i}$ has $n_{i}$ vertices and two vertices are adjacent if and only if they are in distinct parts. If $n_{1}=n_{2}=\ldots=n_{p}$, the graph is regular [7].

Theorem 9.[9] Let $p \geq 2$ be an integer. If $G$ is a p-partite graph of order $n$ such that

$$
n \leq \delta(G) \frac{2 p-1}{2 p-3}
$$

then $\kappa(u, v)=\min \{\operatorname{deg} u, \operatorname{deg} v\}$ for all pairs of distinct vertices $u$ and $v$ in $G$.

Theorem 10. For any integer $p \geq 3$, let $G$ be a regular complete p-partite graph of order $n$. Then $\chi_{l c}(G)=p$.

Proof. Let $G=K_{n_{1}, n_{2}, \ldots, n_{p}}$ be a regular complete $p$-partite graph with the partition $V(G)=V_{1} \cup V_{2} \cup \ldots \cup V_{p}$ to the independent sets $V_{1}, V_{2}, \ldots, V_{p}$, where $\left|V_{i}\right|=n_{i}$ for each $i$ and $n_{1}=n_{2}=\ldots=n_{p}=t, t \geqslant 2$. Thus, $n_{1}+n_{2}+\ldots+n_{p}=n=t p$ and $\operatorname{deg} u=(p-1) t=\delta(G)$ for all $u \in V(G)$. 
Since

$$
\begin{aligned}
\delta(G) \frac{2 p-1}{2 p-3} & =(p-1) t \frac{2 p-1}{2 p-3} \\
& =(p-1) t\left(1+\frac{2}{2 p-3}\right) \\
& >(p-1) t\left(1+\frac{2}{2 p}\right) \\
& =(p-1) t\left(1+\frac{1}{p}\right) \\
& =\frac{p-1}{p} t(p+1) \\
& =\left(1-\frac{1}{p}\right) t(p+1) \\
& >(p+1) t \\
& >p t=n
\end{aligned}
$$

by Theorem $9, \kappa(u, v)=\min \{\operatorname{deg} u, \operatorname{deg} v\}=(p-1) t$ for all pairs of distinct vertices $u$ and $v$ in $G$.

For $p \geq 3$ and $t \geq 2$,

$$
\kappa(u, v)=(p-1) t>p .
$$

Since every vertex in $V_{i}$ is adjacent to each vertex in $V_{j}$, we have $\chi_{l c}(G) \geq p$, where $i, j \in\{1,2, \ldots, p\}, i \neq j$. Without loss of generality assume that $\chi_{l c}(G) \geq p+1$. Since $G$ is colored with at least $p+1$ colors, $\kappa(u, v)<p$ for a vertex $u$ which receives color $p+1$ and a vertex $v$ which receives color $p$. This contradicts (4). Thus, we have $\chi_{l c}(G)=p$.

\section{Conclusion}

In this paper, we introduce and study a new coloring concept called local connective coloring. Since efficiency and reliability of routing can be achieved by using internally disjoint paths, we use the term internally disjoint path in our definition. We give some general bounds and study on several graph classes. Also, we give relation between local connective coloring and some other colorings.

\section{Competing interests}

The authors declare that they have no competing interests.

\section{Authors' contributions}

All authors have contributed to all parts of the article. All authors read and approved the final manuscript.

\section{References}

[1] A. Dandashi and M.Al-Mouhamed, Graph coloring for class scheduling, In Proceedings of IEEE/ACS International Conference on Computer Systems and Applications (AICCSA), 1-4, 2010. 
[2] A. Vasilyev, R. Darda and D. Stevanovi, Trees of Given Order and Independence Number with Minimal First Zagreb Index, MATCH Communications in Mathematical and in Computer Chemistry, 72, 775-782, 2014.

[3] A. William and S. Roy, Packing Chromatic Number of Cycle Related Graphs, International Journal of Mathematics and Soft Computing, 4, 27-33, 2014.

[4] B. Brešar, S. Klavžar, and D.F. Rall, On the packing chromatic number of Cartesian products, hexagonal lattice, and trees, Discrete Appl. Math. 155, 2303-2311, 2007.

[5] C.N. Lai, Optimal construction of all shortest node-disjoint paths in hypercubes with applications, IEEE Transactions on parallel and Distributed Systems, 23, 1129-1134, 2012.

[6] F. Iqbal and F. A. Kuipers, Disjoint Paths in Networks, Wiley Encyclopedia of Electrical and Electronics Engineering, Wiley, 2015.

[7] G. Chartrand, L. Lesniak, and P. Zhang. Graphs \& Digraphs, Fifth edition, Taylor \& Francis, 2010.

[8] K. Menger, Zur allgemeinen Kurventheorie, Fundementa Mathematicae, 10, 96-115, 1927.

[9] J.Topp and L.Volkmann, Sufficient conditions for equality of connectivity and minimum degree of a graph, Journal of Graph Theory 17, 695-700, 1993.

[10] M. Klešč and Š. Schrötter, On the packing chromatic number of semiregular polyhedra, Acta Electrotechnica et Informatica, 12 , 27-31, 2012.

[11] P. M.Talaván and J.Yáñez, The graph coloring problem: A neuronal network approach, European Journal of Operational Research, 191(1), 100-111, 2008.

[12] R. Qu, E.K. Burke and B. McCollum, Adaptive automated construction of hybrid heuristics for exam timetabling and graph colouring problems, European Journal of Operational Research, 198(2), 392-404, 2009.

[13] T. Park and C.Y. Lee, Application of the graph coloring algorithm to the frequency assignment problem, Journal of the Operations Research society of Japan, 39(2), 258-265, 1996.

[14] W. Goddard, S. M. Hedetniemi, S. T. Hedetniemi, J. M. Harris and D. F. Rall, Broadcast chromatic numbers of graphs, Ars Combinatoria, 86,33-50, 2008. 